\title{
Cybersanté et médecine
}

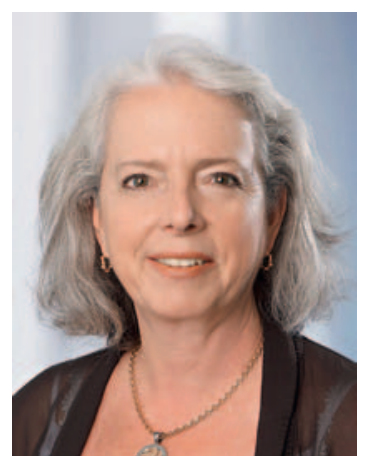

Il y a 16 ans, Health Info Net (HIN) a été fondée à l'initiative de la FMH et de la Caisse des médecins, sur une vision novatrice. HIN avait pour objectif de promouvoir l'échange de données médicales par voie électronique et de manière hautement sécurisée. Rapidement, cette plateforme a connu un essor considérable avec plus de 13500 membres individuels, couvrant ainsi près de $80 \%$ des cabinets médicaux en Suisse et plus de 260 autres acteurs du système de santé, comme les hôpitaux et les soins à domicile.

En 2012, HIN termine sa migration sur une nouvelle plateforme technique, utilisant des standards ouverts, et résolument tournée vers l'avenir avec des moyens d'identification et d'authentification qui peuvent être utilisés avec les outils modernes, ordinateurs, tablettes, health professional card (FMH-HPC) et smartphones. Ainsi, HIN met à disposition de tous les partenaires du domaine de la santé en Suisse une plateforme extranet ouverte et sécurisée pour l'échange de courriers électroniques, de documents et d'autres applications, et ceci grâce à un système robuste, éprouvé, et connu des prestataires. La nouvelle plateforme permet d'ajouter à cette robustesse une réelle simplicité.

\section{II y a 16 ans, HIN a été fondée à l'initia- tive de la FMH et de la Caisse des méde- cins sur une vision avant-gardiste.}

Nous avons donc là un système né d'une vision avantgardiste, largement déployé et utilisé dans le monde de la santé, robuste et simple, basé sur des standards, ouvert aux nouvelles technologies, un système dont la philosophie a toujours été le respect de la sphère privée, ce qui est sans aucun doute le fondement de l'échange de données dans le monde de la médecine. Un tel réseau, dans une époque où l'accès aux données personnelles se banalise, est un préalable indispensable pour que la cybersanté soit utilisée, pour que médecins et patients puissent s'appuyer sur une confiance totale dans la sécurité des échanges et donc dans la protection du secret médical. C'est depuis l'Antiquité la composante centrale de la qualité des soins!

Ces dernières années, toujours plus de projets de cybersanté voient le jour dans toute la Suisse, résultats d'initiatives variées. Que ce soit de grands projets cantonaux, comme le projet genevois eToile, le projet du canton de Bâle ou de Saint-Gall, ou des dossiers partagés commerciaux ou privés, ou encore des initiatives de groupes de médecins ou d'assurances, ces projets utilisent des méthodes diverses de sécurisation et d'authentification. Indépendamment de la robustesse ou de la qualité de ces systèmes, il n'en demeure pas moins vrai qu'ils créent pour le moment une hétérogénéité et donc une complexité qui s'accommode mal avec les besoins quotidiens des médecins.

\section{Un tel réseau est indispensable pour que médecins et patients puissent s'appuyer sur une confiance totale dans la sécurité des échanges.}

Ne serait-il pas souhaitable d'utiliser HIN comme pierre angulaire de la sécurisation des projets cybersanté en Suisse? Le système est là, le besoin aussi! Il faut montrer à nos autorités et à tous les initiateurs de projets de cybersanté que, certes, les outils de cybersanté peuvent nous simplifier la vie, améliorer la qualité des soins, mais seulement s'ils sont intégrés dans la pratique quotidienne, et seulement s'ils utilisent des systèmes déjà en place et cohérents. Et que les solutions ne seront pas utilisées si elles s'appuient sur de nouveaux systèmes, qui complexifient leurs tâches quotidiennes. Il faut sûrement que nous, médecins, nous luttions pour qu'une solution comme HIN soit au centre de notre système suisse de cybersanté!

Dr Monique Gauthey, médecin spécialiste FMH, membre du Comité central de la FMH, responsable du domaine eHealth - infrastructure de sécurité 